\title{
Recurrent Larynx and Hypopharyngeal Liposarcoma with Systemic Progression
}

\section{Kuauhyama Luna-Ortiz ${ }^{1,2 *}$, Roberto Rodriguez-Garcia ${ }^{1}$ and Horacio N Lopez Basave}

${ }^{1}$ Department of Head and Neck Surgery, Instituto Nacional de Cancerologia A, San Fernando, Tlalpan, Mexico

${ }^{2}$ Departament of General Surgery (Head and Neck), Hospital Manuel Gea Gonzalez, Mexico

${ }^{3}$ Department of Gastroenterology Instituto Nacional de Cancerologia A, Mexico

\begin{abstract}
Introduction: Liposarcomas are infrequent tumors. Only $5.6 \%$ of these tumors occur in the head and neck. Primary liposarcomas of the larynx comprise $\sim 10 \%$ to $15 \%$ of head and neck liposarcomas. Multiple surgeries and recurrences history are common.

Case summary: We present the case of a 33-year-old male whose illness started at 16 years of age, and who underwent multiple surgeries due to multiple recurrence (six) by a liposarcoma in the neck, all treated with organ preservation. In 2014 patient arrived with a big recurrence a total pharyngolaryngectomy with a jejunal are flap was performed. after 18 month of the follow up the patient comes to the visits and refered developed headache, ataxia and shortness of breath, that is why a MRI of the brain and CT of the torax shown intraxial lesions in the brain and multiple bilateral pulmonary nodules compatible with metastatic disease, We propose palliative treatment with radiotherapy but the patient refuse treatment so he was sent to palliative care.
\end{abstract}

Discussion: The mainstay of treatment is surgical resection. Multiple recurrence is the rule but metastasis to the brain was not reported before. The role of radiotherapy is not well know. Chemotherapy until now showed no sustancial benefit.

Keywords: Head and neck sarcomas; Liposarcoma; Metastasis; Brain; Recurrence

\section{Introduction}

Liposarcomas are malignant mesenchymal tumors that most frequently occur in the extremities and retroperitoneum. Only $5.6 \%$ of these tumors occur in the head and neck area. Most of these originate in the soft tissues of the neck [1,2]. The first reports of head and neck liposarcomas were described during the 1960s with liposarcomas of the hypopharynx being extremely rare. Approximately 38 cases have been reported in the English, French and Spanish literature [3,4]. Primary liposarcomas of the larynx comprise $\sim 10 \%$ to $15 \%$ of head and neck liposarcomas [5]. Approximately $60 \%$ of the cases occur in males. Mean age of presentation is between the $4^{\text {th }}$ and $6^{\text {th }}$ decades of life. Symptom presentation is mainly dysphagia and dyspnea $[3,6-8]$. We present the case of a young male patient who underwent multiples surgeries and recurrences until being subjected to extensive surgery with jejunal free flap reconstruction and final metastasis disease to the brain.

\section{Case Report}

A 33-year-old male whose illness initiated at 16 years of age with dysphonia and dysphagia due to a tumor originating from the right piriform sinus and treated with two transcervical resections and diagnosis of myxoid liposarcoma. In 2005, the patient was referred to us for recurrence, a transoral surgical resection with $\mathrm{CO}_{2}$ laser was performed, and surgical margins were negative. A year later, a recurrence and surgery was proposed and refused by the patient. Patient was lost of follow-up for 8-year, during that two additional transcervical resections were performed. In november 2014 he returned to our institution due to a $6^{\text {th }}$ new recurrence. He presented with dysphagia to fluids and significant dysphonia with laryngeal stridor and dyspnea on minimal effort. Magnetic resonance imaging (MRI) demonstrated a tumor extending from the piriform sinus and right lateral hypopharyngeal wall to the paraglottic space, with massive infiltration of the larynx with no invasion to tongue base (Figures 1a-1f). A total pharyngolaryngectomy and reconstruction with a jejunal free graft. A tumor of $10 \mathrm{~cm} \times 12 \mathrm{~cm}$ with epicenter in the right hypopharynx (Figures 2a-2d). Patient was discharged on the tenth postoperative day with a gastrostomy tube

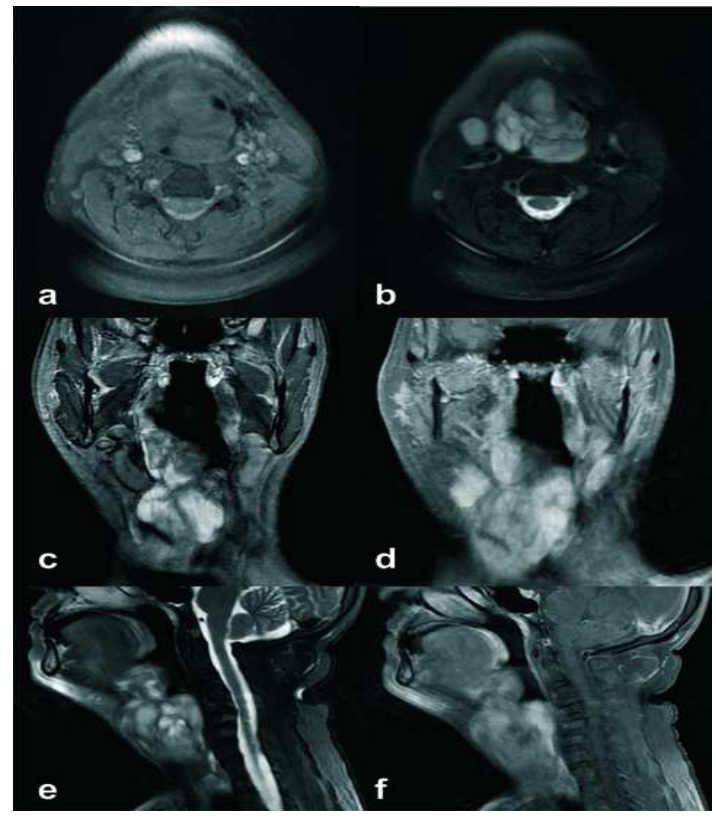

Figure 1: Gadolinium-enhanced MRI images which show a hyperintense lesion whose epicenter is located in the right hemilarynx $(a, b)$, with major invasion to the right lateral wall of the hypopharynx $(c, d)$, right paraglottic space and preepiglotic space (d,e and f).

*Corresponding author: Kuauhyama Luna-Ortiz, MD, Department of Head and Neck Surgery, Instituto Nacional de Cancerologia, San Fernando, Tlalpan, Mexico, Tel: 52556280400; E-mail: kuauhyama@yahoo.com.mx

Received November 03, 2017; Accepted December 26, 2017; Published December 29, 2017

Citation: Luna-Ortiz K, Rodriguez-Garcia R, Basave HNL (2017) Recurrent Larynx and Hypopharyngeal Liposarcoma with Systemic Progression. J Clin Case Rep 7: 1060. doi: $10.4172 / 2165-7920.10001060$

Copyright: (๑) 2017 Luna-Ortiz K, et al. This is an open-access article distributed under the terms of the Creative Commons Attribution License, which permits unrestricted use, distribution, and reproduction in any medium, provided the original author and source are credited. 


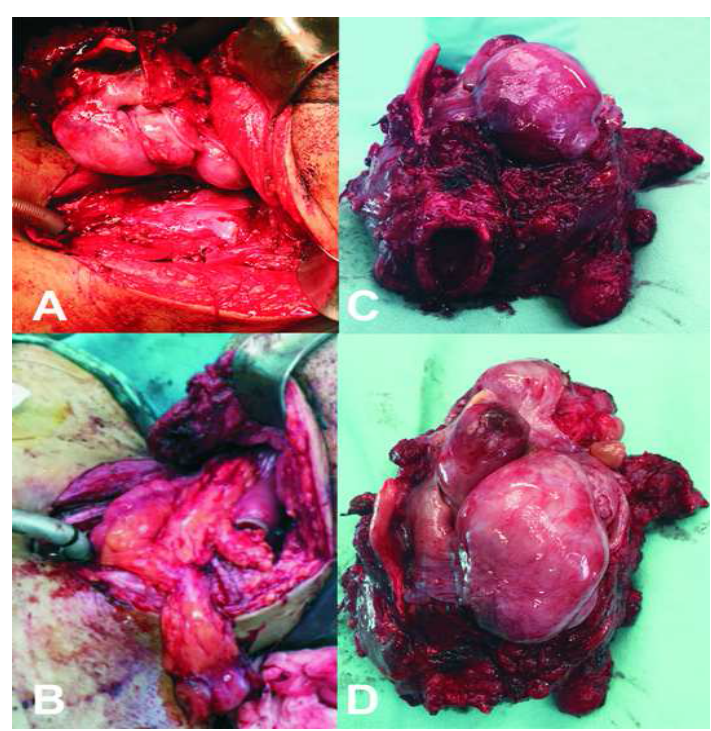

Figure 2: (a,c,d) Surgical specimen demonstrates a right hemilarynx based neoplastic lesion, with evident invasion to paraglottic space and right wal of the hypopharynx and minor invasion to preepiglotic space. (b). Inset of microvascular jejunal free flap to be anastomosed to remaining portion hypopharynx and cervical esophagus.

feeding and no complications. A myxoid liposarcoma with round cells component $(20 \%)$ and lymphovascular invasion and margins were negative. Adjuvant radiotherapy was proposed but patient refuse. At 18 month of the follow up the patient refered headache, ataxia and shortness of breath, a MRI of the brain and CT of the torax shown intraxial lesions to the brain and multiple metastasis to the lung. We propose palliative treatment with radiotherapy but the patient refuse any treatment so he was sent to palliative care.

\section{Discussion}

Liposarcomas of the hypopharynx are rare tumors accounting for only $3 \%$ to $8 \%$ of liposarcomas presenting in the head and neck. These generally occur in the soft tissues of the neck [9]. There have been $\sim 30$ cases of this type of tumor in the hypopharynx with a male: female ratio of 9:1 [4]. Risk factors for developing this type of tumor are unknown. Gerry et al. [9] report that the combined incidence in the larynx and hypopharynx was $6 \%$. This study also reveals that the biologic behavior of head and neck liposarcomas is more favorable than those of the trunk. There are up to seven different histological subtypes of this malignancy reported in the literature. Of these, myxoid liposarcoma continues to be the most common in the head and neck region with $\sim 79 \%$ of the cases. Our patient had mostly a myxoid component; however, areas of rounded cells were present in $20 \%$ of the fields studied in the surgical specimen [9].

The most common clinical presentation is associated with obstructive symptoms of the aerodigestive tract such as dysphagia and dysphonia. Lymph node involvement at the time of diagnosis is infrequent.

There is no standardized surgical management for these tumors due to their infrequent presentation, but surgery is currently the cornerstone of treatment without real evidence on the role of radiotherapy. This is demonstrated in the study by Gerry et al. [9] where $79.6 \%$ of the patients were treated exclusively with surgery for head and neck tumors. The anatomic location makes it very difficult to perform surgery with wide margins in comparison with other locations such as the trunk or extremities due to the closeness of neural and vascular structures which, if resected, are associated with significant morbidity. These tumors are associated with a high rate of recurrence especially when organ preservation is attempted. One of the main prognostic factors in the treatment of sarcomas is the status of the resection margins. In the literature, surgical management varies in different case reports from management using marginal resection, transoral laser surgery, and wide resections $[1,3,5,7,8,10]$. All series were in agreement and the case reports indicate a high rate of local recurrence with locally aggressive behavior and, rarely, distant disease, there is not report in the literature with metastases to the brain $[5,8]$. There is not any randomized control trial in the literature who shows sustancial benefit in the adittion of adyuvant chemotherapy.

\section{Conclusion}

The mainstay of treatment is surgical resection. Multiple recurrence is the rule but metastasis to the brain was not reported before. The role of radiotherapy is not well known.

\section{References}

1. Gritli S, Khamassi K, Lachkhem A, Touati S, Chorfa A, et al. (2010) Head and neck liposarcomas. A 32-year experience. Auris Nasus Larynx 37: 347-351.

2. Burningham Z, Hashibe M (2012) The epidemiology of sarcoma. Clin Sarcoma Res 2: 14.

3. Luna-Ortiz K, Campos-Ramos E, Carmona-Luna T, Mohar-Betancourt A Ferrari-Carballo T (2009) Laser resection of liposarcoma of the hypopharynx. Med Oral Pathol Cir Bucal 14: E252-256.

4. Mestre de Juan JM, Fernández Acenero MJ (1999) Laryngeal liposarcoma Report of a case. Pathol Res Pract 195: 125-128.

5. Han Y, Ting-Ting L (2014) Liposarcoma of the larynx: Report of a case and review of the literature. Int J Clin Exp Pathol 8: 1068-1072.

6. Wenig B, Heffner DK (1995) Liposarcomas of the larynx and hypopharynx A clinicopathologic study of eight new cases and a review of the literature. Laryngoscope 105: 747-756.

7. Sotiróvic J, Vukomanovic-Djurdevic B, Baletic N, Pavicevic L, Bijelic D, et al. (2014) Recurrent lipomatous tumor of hypopharynx: Case report literature review. Acta Clin Croat 53: 365-368.

8. Sanz-Gonzalo J, Martínez-Molina P, Ribalta Farres MT, Babaetr Matadelabarata F (2002) Liposarcoma de hipofaringe. Acta Otorrinolaringol Esp 53: 60-63.

9. Gerry DL, Fox NF, Spruill LS, Lentsch EJ (2014) Liposarcoma of the head and neck: Analysis of 318 cases with comparison to non-head and neck sites. Head Neck 36: 393-400.

10. Kodiyan J, Rudman J, Rosow D, Thomas G (2015) Lipoma and liposarcoma of the larynx: Case reports and literature review. Am J Otolaryngol 36: 611-615. 